\title{
Post-stenotic coronary blood flow at rest is not altered by therapeutic doses of the oral antidiabetic drug glibenclamide in patients with coronary artery disease
}

\author{
T Reffelmann, H G Klues, P Hanrath, E R Schwarz
}

Heart 2002;87:54-60

See end of article for authors' affiliations

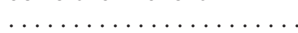

Correspondence to: Dr T Reffelmann, Medizinische Klinik I, University Hospital, Rheinisch-Westfälische Technische Hochschule (RWTH), Pauwelsstrasse 30, D-52057 Aachen, Germany;

thorstenreffelmann@web.de

Accepted 22 August 2001

\begin{abstract}
Objective: To investigate whether blood flow in normal and post-stenotic coronary arteries is altered by therapeutic doses of the sulfonylurea agent glibenclamide.

Patients: 12 patients with a high grade stenosis of the left anterior descending coronary artery $(n=10)$ or left circumflex coronary artery $(n=2)$, and an angiographically normal corresponding left circumflex artery or left anterior descending artery, respectively.

Design: Two Doppler ultrasound wires were positioned in the "normal" and post-stenotic artery for simultaneous measurements of coronary blood flow velocity under baseline conditions and after intravenous glibenclamide, $0.05 \mathrm{mg} / \mathrm{kg}$ body weight. Local coronary blood flow was calculated from the average peak velocity and the cross sectional area derived from quantitative coronary angiographic analysis. Coronary flow reserve was determined after intracoronary injection of $30 \mu \mathrm{g}$ adenosine and $12 \mathrm{mg}$ papaverine.

Results: One hour after glibenclamide, serum insulin increased from (mean (SD)) 7.4 (2.0) to 44.8 (25.5) $\mathrm{mU} / \mathrm{I}(\mathrm{p}<0.005)$, and $C$ peptide from $1.4(0.4)$ to 3.4 (1.2) $\mathrm{ng} / \mathrm{I}$ ( $\mathrm{p}=0.005)$. In normal coronary arteries coronary flow reserve was $2.6(0.4)$ after adenosine and $3.0(0.4)$ after papaverine, while in post-stenotic arterial segments it was $1.2(0.3)$ after adenosine $(p=0.005)$ and $1.3(0.3)$ after papaverine $(p=0.005)$. There was no significant difference after glibenclamide. In non-stenotic arteries, average peak velocity $(18.8(5.2) \mathrm{cm} / \mathrm{s})$ and calculated coronary blood flow $(23.8(10.7) \mathrm{ml} / \mathrm{min})$ were not altered by glibenclamide $(18.3(5.2) \mathrm{cm} / \mathrm{s}$ and $22.8(10.4) \mathrm{ml} / \mathrm{min}$, respectively). In post-stenotic arteries, baseline average peak velocity was $13.3(4.9) \mathrm{ml} / \mathrm{min}$ and coronary blood flow was 9.1 (3.0) $\mathrm{ml} / \mathrm{min}$, without significant change after glibenclamide (13.3 (5.2) cm/s, 9.0 (3.2) ml/ $\min )$.

Conclusions: Glibenclamide, $0.05 \mathrm{mg} / \mathrm{kg}$ intravenously, is effective in increasing serum insulin, suggesting a $\mathrm{K}_{\text {ATP }}$ channel blocking effect in pancreatic $\beta$ cells. It does not compromise coronary blood flow and vasodilatation in response to adenosine and papaverine in post-stenotic and angiographically normal coronary arteries at rest.
\end{abstract}

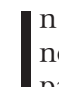
$\mathrm{n}$ the USA, approximately six million people suffer from non-insulin dependent diabetes mellitus ( $85 \%$ of all diabetic patients), and are potentially eligible for treatment with sulfonylurea agents. ${ }^{1}$ Sulfonylurea drugs inhibit potassium efflux through ATP sensitive potassium channels $\left(K_{\text {ATP }}\right.$ channels) in pancreatic $\beta$ cells. This results in increased exocytic insulin release. ${ }^{2} \mathrm{~K}_{\text {ATP }}$ channels have been found in several tissues-for example, cardiac myocytes, vascular smooth muscle cells, in the central nervous system, and in the kidney. Thus complex effects on different organ systems in patients with sulfonylurea treatment need to be considered during follow up.

In several animal models, hypoxic dilatation of coronary arteries has been shown to be mediated by activation of vascular $\mathrm{K}_{\text {ATP }}$ channels. ${ }^{3}$ The role of these channels in the coronary circulation has been investigated in various experimental settings. It has been suggested that they contribute to the control of basal coronary tone and coronary vasodilatation in response to adenosine.$^{3-5}$ In dogs, a synergistic role of adenosine and $\mathrm{K}_{\text {ATP }}$ channels in maintaining coronary flow distal to an artificial stenosis has been demonstrated. ${ }^{6}$ Thus a potential effect of $\mathrm{K}_{\text {ATP }}$ channel blocking agents, such as the antidiabetic sulfonylurea drugs, on the coronary circulation in different physiological and pathophysiological conditions is a matter of debate. $^{7}$ However, the effects of sulfonylurea drugs on coronary blood flow in patients with coronary artery disease have not yet been clarified.
Cardiovascular mortality in diabetic patients on sulfonylurea drugs may be increased in comparison with those on insulin treatment. ${ }^{8}$ In comparison with insulin treated patients, an increased mortality has recently been reported in patients on sulfonylurea drug treatment undergoing primary angioplasty for acute myocardial infarction.

It is suggested that opening of the cardiac $\mathrm{K}_{\mathrm{ATP}}$ channels is involved in the phenomenon of ischaemic preconditioning, in which a notable reduction in infarct size occurs if the myocardium has been pretreated with brief episodes of ischaemia followed by reperfusion. The increased mortality during treatment with sulfonylurea agents has mainly been attributed to inhibition of "preconditioning-like" effects, resulting in a reduced cardioprotective potential. ${ }^{10}$ In human patients, however, this has not yet been proven. Theoretically, the blockade of coronary $\mathrm{K}_{\text {AтP }}$ channels by sulfonylurea drugs might compromise coronary blood flow. For example, it has been shown that the forearm vasodilator response to brief periods of arterial occlusion is reduced after pharmacological blockade of $\mathrm{K}_{\text {ATP }}$ channels. ${ }^{11}$ This possible interaction between sulfonylurea agents and vascular $K_{\text {ATP }}$ channels is expected to occur at therapeutic doses of the drug. Although the plasma concentrations of sulfonylurea required to block cardiac and vascular $\mathrm{K}_{\text {ATP }}$ channels are higher than those inducing pancreatic insulin release, concentrations of glibenclamide that are effective at pancreatic $\beta$ cells could also affect a proportion of the vascular $\mathrm{K}_{\text {ATP }}$ channels. ${ }^{12}$ 
Our aim in the present study was to investigate the effects of therapeutic doses of glibenclamide on coronary blood flow in patients with coronary artery disease. We hypothesised that a therapeutic dose of glibenclamide could alter post-stenotic coronary blood flow or vasodilatation in response to adenosine. We studied the influence of glibenclamide on coronary flow in angiographically normal coronary arteries and arteries with a high grade stenosis using intracoronary Doppler flow measurements and quantitative coronary angiography.

\section{METHODS}

Written informed consent was obtained from all the patients 24 hours before they were included in the study. The study protocol was approved by the local institutional ethics review board.

\section{Patient selection}

We studied 12 patients (mean (SD) age, 54 (6) years; six female, six male) with a high grade stenosis ( $>90 \%$ diameter stenosis) of the left anterior descending coronary artery or the left circumflex artery (or a major branch). After diagnostic coronary angiography the patients were scheduled for angioplasty because of significant ST segment depression or terminal $\mathrm{T}$ wave inversion during or after bicycle testing (four patients), objective signs of ischaemia on myocardial scintigraphy (five patients), or reproducible symptoms of angina during exercise testing (seven patients).

Patients were included in the study if there was a high grade stenosis of the left anterior descending coronary artery (10 patients) with an angiographically normal left circumflex coronary artery, or a high grade stenosis of the left circumflex artery (two patients) with a "normal" left anterior descending artery.

Exclusion criteria were unstable angina, acute myocardial infarction (less than four weeks before), atrial fibrillation, second or third degree atrioventricular block, left or right bundle branch block, valvar heart disease, cardiomyopathy, chronic renal failure (serum creatinine $>177 \mu \mathrm{mol} / \mathrm{l}$ ), a history of allergic reactions to sulfonylurea agents or sulfonamides, previous treatment with sulfonylureas, and oral anticoagulation treatment.

All cardiovascular drugs, including $\beta$ receptor blockers, calcium antagonists, long acting nitrates, and angiotensin converting enzyme inhibitors, were withheld 24 hours before the procedure. Where there were anginal symptoms, patients were treated with short acting nitrates.

\section{Coronary angiography and quantitative diameter measurements}

Cardiac catheterisation was performed routinely by the Judkins technique with 7 or 8 French catheters through a femoral sheath (contrast agent: iopromide, Ultravist-370, Schering AG, Berlin, Germany). Quantitative measurements of the coronary artery diameters and left ventricular ejection fraction were obtained from digitised cineangiograms, using the coronary angiography analysis system II (Pie Medical, Maastricht, Netherlands). Details of this edge detection based method have been published previously. ${ }^{13}$ No intracoronary glyceryl trinitrate was given, as this might interfere with the measured vessel diameters and blood flow velocities. The percentage diameter stenosis and the average lumen diameters in two orthogonal projections $5 \mathrm{~mm}$ distal to the tip of the Doppler wire were measured, using the guiding catheter as a scaling device along with a user defined reference lumen diameter of an angiographically normal coronary artery segment.

\section{Intracoronary Doppler flow velocity measurements} Intracoronary Doppler flow profiles were obtained using a 0.014 inch $(0.36 \mathrm{~mm})$ flexible Doppler guide wire (12 MHz, FloWire, Cardiometrics Inc, Mountain View, California, USA).
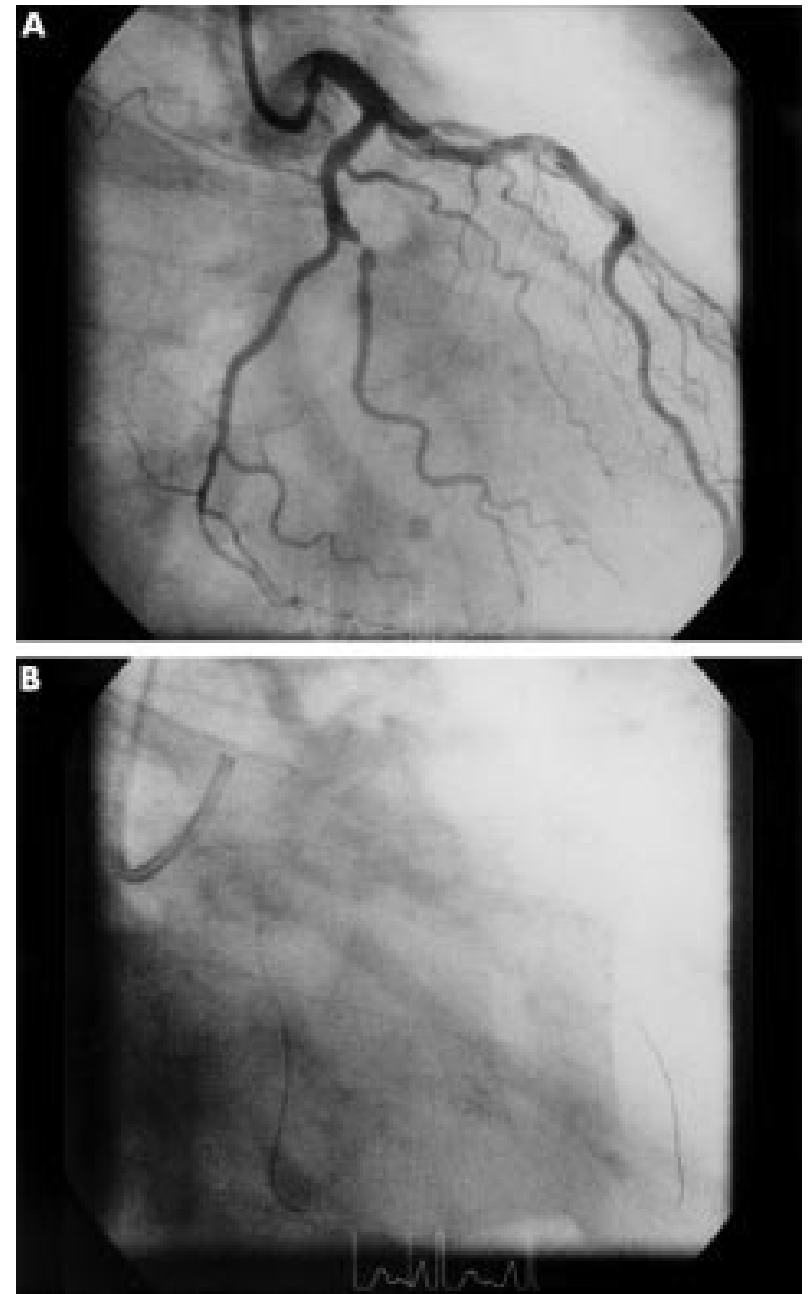

Figure 1 Original coronary angiographic images of a patient with a high grade stenosis of the marginal branch (A). During the procedure, one Doppler flow wire was positioned in the distal left anterior descending coronary artery and a second in the distal marginal branch distal to the stenosis (B) (7 French guiding catheter). Suitable projections were chosen to measure the degree of the stenosis and thereafter to determine coronary vessel diameters in two orthogonal projections $5 \mathrm{~mm}$ distal to the tip of the flow wire.

After stable placement of the guiding catheter in the left coronary ostium, one Doppler guide wire was positioned in the distal left anterior descending coronary artery and the other in the distal left circumflex coronary artery for simultaneous measurements of coronary flow velocity. A switch box allowed measurement of Doppler flow profiles in both coronary arteries without disconnecting the wires. The wire in the coronary artery with the high grade stenosis was positioned distal to the stenosis (fig 1). Data were obtained after we had confirmed that the position of both wires was stable by fluoroscopic imaging and flow measurements over a period of three minutes. Throughout the procedure, average peak velocity, average systolic peak velocity, and average diastolic peak velocity (all in $\mathrm{cm} / \mathrm{s}$ ) were recorded in the coronary artery segments.

In both coronary arteries, coronary flow reserve was determined by intracoronary injection of $30 \mu \mathrm{g}$ of adenosine and $12 \mathrm{mg}$ of papaverine. Coronary flow reserve was calculated as the maximum average peak velocity (after adenosine or papaverine) divided by the baseline average peak velocity.

\section{Quantitative calculation of local coronary blood flow}

During the experimental procedure the average peak flow velocity was recorded on-line in both coronary vessels. When 
Table 1 Clinical and angiographic characteristics of the 12 patients with coronary artery disease

\begin{tabular}{ll}
\hline Patients & 12 \\
$n$ & $6 / 6$ \\
Female/male & $54(6)$ \\
Age (years) & \\
& \\
Angiographic characteristics & $56(9) \%$ \\
Ejection fraction & $94(5) \%$ \\
Diameter stenosis (10 LAD, 2 LCx) & 4 \\
Additional stenosis of the right coronary artery & \\
& \\
Cardiovascular risk factors & 8 \\
Systemic hypertension & 7 \\
Hypercholesterolaemia & 5 \\
History of cigarette smoking or current smoker & 0 \\
Diabetes mellitus & 8 \\
Obesity & 6 \\
Family history of cardiovascular diseases & \\
\hline Values are mean (SD). & \\
LAD, left anterior descending coronary artery; LCx, left circumflex \\
coronary artery.
\end{tabular}

Doppler flow profiles were obtained, biplane coronary angiography was done in two orthograde projections. Vessel diameters were measured $5 \mathrm{~mm}$ distal to the tip of the wire. Vessel cross sectional area (CSA) and local coronary blood flow $(\mathrm{CBF})$ were calculated as follows ${ }^{14}{ }^{15}: \mathrm{CSA}=0.25 \times \mathrm{D}_{\mathrm{RAO}} \times \mathrm{D}_{\mathrm{LAO}}$ $\times \pi$, where $\mathrm{D}_{\mathrm{RAO}}, \mathrm{D}_{\mathrm{IAO}}=$ mean vessel diameter in right anterior oblique and left anterior oblique projections; and $\mathrm{CBF}=\mathrm{CSA}$ $\times 0.5 \times$ average peak flow velocity.

\section{Measurements of serum insulin, $\mathrm{C}$ peptide, and blood glucose}

Before the catheterisation procedure and one hour after infusion of glibenclamide $(0.05 \mathrm{mg} / \mathrm{kg}$ body weight $)$, serum samples were obtained for analysis of insulin and $\mathrm{C}$ peptide. The samples were analysed by immunometric assay and competitive immunoassay (Immulite Insulin/Immulite C Peptide, Immulite Analyser, DPC Diagnostic Products Corp, Los Angeles, California, USA). The central 95\% reference range for insulin was 6-27 mU/l, and for C peptide, $0.3-1.3 \mathrm{nmol} / \mathrm{l}$.

Blood glucose was measured by bedside testing (Glucometer Elite 3906, Bayer Diagnostics, Munich, Germany) directly before the infusion of glibenclamide and then at 30 minute intervals.

\section{Drugs}

The following drugs were used during the catheterisation procedure:

- glibenclamide (HB 419) (lyophilisate for parenteral use), a gift from Hoechst AG, Frankfurt, Germany; the drug was dissolved in $15 \mathrm{ml}$ sterile $0.9 \% \mathrm{NaCl}$ directly before infusion

- papaverine (Paveron, Linden, Heuchelheim, Germany)

- adenosine (Adrekar, Sanofi Winthrop, Munich, Germany).

\section{Experimental protocol}

Four hours before the procedure, an intravenous infusion of $5 \%$ glucose at a rate of $60 \mathrm{ml} / \mathrm{h}$ was initiated and continued for 24 hours. After insertion of the femoral sheath catheter and intra-arterial administration of $140 \mathrm{IU}$ heparin $/ \mathrm{kg}$ body weight, biplane coronary angiography of the left coronary artery was performed for selection of optimal fluoroscopic projections. Under fluoroscopic control, one Doppler wire was placed in the left anterior descending coronary artery and a second was placed in the left circumflex coronary artery. In the coronary artery with the high grade stenosis, the wire was placed distal to the stenosis. After recording the baseline Doppler indices (three independent measurements over a period of three minutes) in both coronary arteries, coronary
Table 2 Coronary flow reserve (CFR) after $30 \mu \mathrm{g}$ adenosine and $12 \mathrm{mg}$ papaverine at baseline and after $0.05 \mathrm{mg} / \mathrm{kg}$ glibenclamide

\begin{tabular}{|c|c|c|c|c|}
\hline & \multicolumn{2}{|c|}{$\begin{array}{l}\text { Poststenotic coronary } \\
\text { artery }\end{array}$} & \multicolumn{2}{|c|}{$\begin{array}{l}\text { Angiographically normal } \\
\text { artery }\end{array}$} \\
\hline & Baseline & $\begin{array}{l}\text { After } \\
\text { glibenclamide }\end{array}$ & Baseline & $\begin{array}{l}\text { After } \\
\text { glibenclamide }\end{array}$ \\
\hline $\mathrm{CFR}_{\text {adenosine }}$ & $1.2(0.3)$ & $1.2(0.2)$ & $2.6(0.4)$ & $2.6(0.3)$ \\
\hline CFR & $1.3(0.3)$ & $1.3(0.3)$ & $3.0(0.4)$ & $2.9(0.4)$ \\
\hline
\end{tabular}

angiography was done in two orthogonal projections. Thereafter, coronary flow reserve was measured after an intracoronary injection of $30 \mu \mathrm{g}$ adenosine and $12 \mathrm{mg}$ papaverine.

After a second recording of baseline Doppler flow profiles (three measurements) with subsequent angiography, glibenclamide $0.05 \mathrm{mg} / \mathrm{kg}$ body weight was given intravenously over a period of 15 minutes. Thereafter, measurements of Doppler indices (three independent measurements) with subsequent coronary angiography were repeated, followed by recording of coronary flow reserve after adenosine and papaverine and a further recording of Doppler flow profiles with subsequent coronary angiography.

\section{Statistical analysis}

Values are given as mean (SD). For comparison of serum insulin and $\mathrm{C}$ peptide concentrations before and after glibenclamide, a paired Student's $t$ test was used. Doppler measurements and haemodynamic and angiographic measurements at different time points were compared by analysis of variance for repeated measurements. Correlation between the grade of the stenosis and coronary flow reserve was determined by the Spearman correlation coefficient. A probability value of $\mathrm{p}<0.05$ was considered significant.

\section{RESULTS}

\section{Patient characteristics}

Twelve patients (mean (SD) age 54 (6) years) were included in the study. Ten patients had a high grade stenosis of the left anterior descending coronary artery and two had a high grade stenosis of the left circumflex coronary artery. Cineventriculographic analysis showed wall motion abnormalities in the corresponding myocardial territory in five patients (moderate hypokinesia in three, severe hypokinesia in two). No patient had a history of myocardial infarction. The ECG did not show $\mathrm{Q}$ waves in the corresponding leads. Demographic, clinical, and angiographic data on the patients are shown in table 1. None of the patients was diabetic and therefore none had ever been on treatment with sulfonylurea drugs.

\section{Stability of the measured variables}

The mean standard deviation of three independent measurements of the average peak velocity (over a three minute period at baseline) was $2.1 \mathrm{~cm} / \mathrm{s}$ (range $0.6-4.1 \mathrm{~cm} / \mathrm{s}$ ). Before measurements of coronary flow reserve, recording of the Doppler profile in the post-stenotic artery revealed an average peak velocity of $13.4(4.8) \mathrm{cm} / \mathrm{s}$. After determination of coronary flow reserve in the post-stenotic artery, baseline average peak velocity was $13.3(4.9) \mathrm{cm} / \mathrm{s}$ (NS $v$ the first baseline recording). In the normal coronary arteries, average peak velocity before and after measurements of coronary flow reserve was also unchanged, at $18.7(5.2) \mathrm{cm} / \mathrm{s}$ and 18.8 $(5.2) \mathrm{cm} / \mathrm{s}$, respectively. No intracoronary glyceryl trinitrate was given during the protocol. Stability patterns were similar after administration of glibenclamide.

Determination of vessel diameters before and after measurements of coronary flow using quantitative coronary angiography showed minimal variation in the measured variables- 

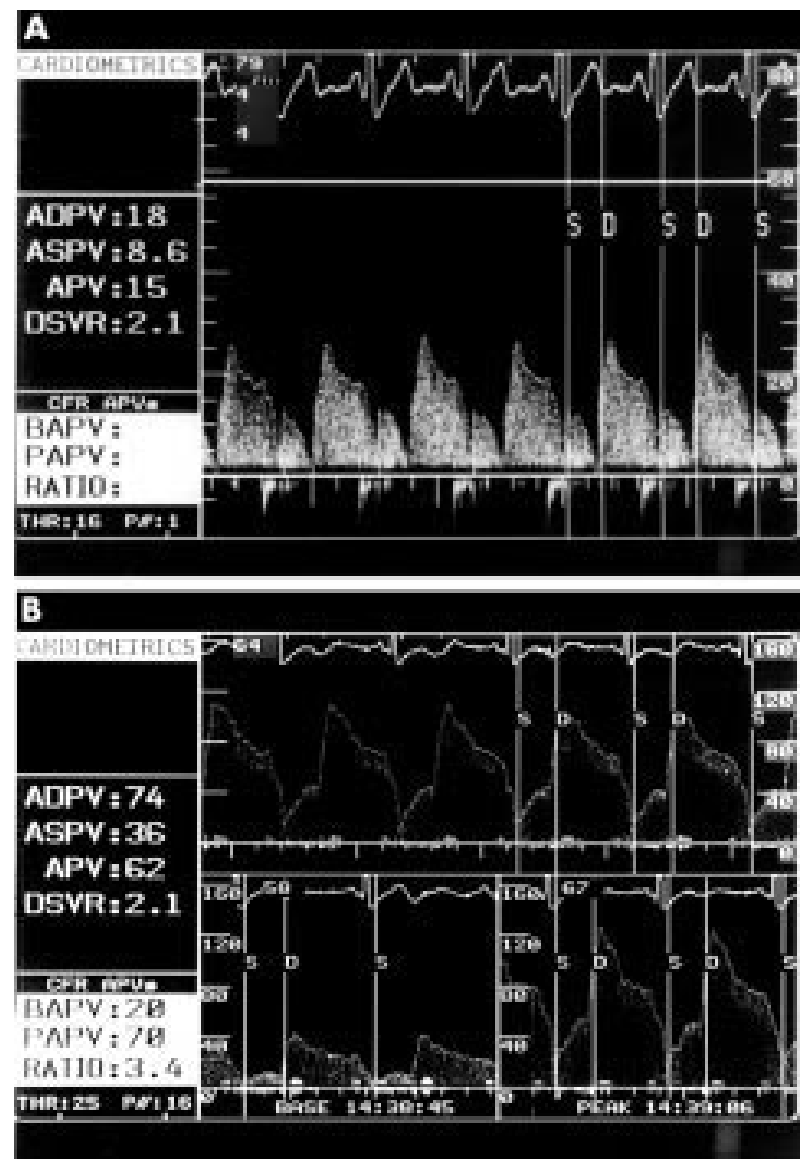

Figure 2 Original Doppler profile at rest $(\mathrm{A})$ and during determination of coronary flow reserve by administration of papaverine in a non-stenotic coronary artery (B). The profiles show the typical increase in diastolic flow velocity. Analysis of the Doppler derived velocity indices was performed by the integrated software throughout the experiments.

for example, the mean cross sectional area was $2.4(0.7) \mathrm{mm}^{2}$, as calculated from the first angiographic image before flow reserve measurements, and $2.4(0.6) \mathrm{mm}^{2}$ in the second image after determination of coronary flow reserve.

Coronary flow reserve after adenosine and papaverine Coronary flow reserve after intracoronary administration of $30 \mu \mathrm{g}$ adenosine was $1.2(0.3)$ in the post-stenotic segment, while in the angiographically normal arteries it was significantly higher, at $2.6(0.4)(\mathrm{p}<0.005)$. After administration of

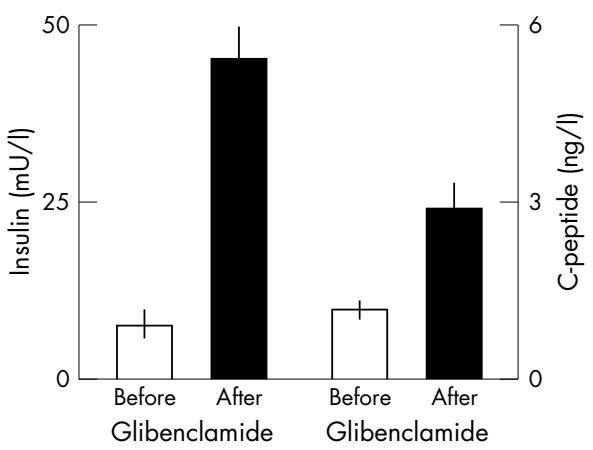

Figure 3 Serum insulin (left) and serum $C$ peptide concentrations (right) before and after administration of glibenclamide, $0.05 \mathrm{mg} / \mathrm{kg}$ body weight. Values are means, error bars $=S D$.

glibenclamide, mean coronary flow reserve did not change, remaining at $1.2(0.2)$ in the post-stenotic segment and 2.6 (0.3) in the normal coronary artery (table 2). Adenosine induced coronary vasodilatation was not significantly altered after glibenclamide. Coronary flow reserve after administration of $12 \mathrm{mg}$ papaverine tended to be higher than after adenosine induced hyperaemia. Coronary flow reserve determined by papaverine did not change before and after glibenclamide (table 2). There was a significant negative correlation between the degree of maximum diameter stenosis and coronary flow reserve after both adenosine and papaverine (Spearman correlation coefficients, -0.7711 for adenosine $(p<0.05)$ and -0.8840 for papaverine $(p<0.005))$. Adenosine and papaverine cause coronary vasodilatation by different mechanisms, papaverine having a direct relaxing effect on vascular smooth muscle cells. Because of this, the coronary flow reserve was always higher after papaverine than after adenosine.

\section{Doppler profiles, vessel diameters, and coronary angiography}

An original Doppler profile, with its typical diastolic increase in flow, is shown in fig 2 . In the post-stenotic artery average peak velocity and vessel size were smaller than in the normal coronary artery (table 3 ). Calculated cross sectional area of the post-stenotic artery was $58 \%$ of that in the "normal" coronary artery, as the measurements were performed in the arterial segment distal to the vessel narrowing. In neither the post-stenotic coronary artery nor the normal coronary artery was there a significant change in average peak velocity, cross sectional area, or calculated quantitative coronary blood flow before or after glibenclamide. Heart rate and systemic blood

Table 3 Flow variables and angiographic measurements in the angiographically normal coronary artery and the high grade stenosis coronary artery, at baseline and after administration of glibenclamide, $0.05 \mathrm{mg} / \mathrm{kg}$ body weight

\begin{tabular}{|c|c|c|c|c|}
\hline & \multicolumn{2}{|c|}{ Poststenotic coronary artery } & \multicolumn{2}{|c|}{ Angiographically normal artery } \\
\hline & Baseline & After glib & Baseline & After glib \\
\hline Mean BP $(\mathrm{mm} \mathrm{Hg})$ & $98.9(7.0)$ & $98.3(7.6)$ & $98.9(7.0)$ & $98.3(7.6)$ \\
\hline Heart rate (beats/min) & $68.6(8.9)$ & $67.0(8.1)$ & $68.6(8.9)$ & $67.0(8.1)$ \\
\hline $\operatorname{APV}(\mathrm{cm} / \mathrm{s})$ & $13.3(4.9)$ & $13.3(5.2)$ & $18.8(5.2)$ & $18.3(5.2)$ \\
\hline $\operatorname{ASPV}(\mathrm{cm} / \mathrm{s})$ & $7.6(2.9)$ & $8.1(3.5)$ & $12.3(3.5)$ & $11.7(3.4)$ \\
\hline $\operatorname{ADPV}(\mathrm{cm} / \mathrm{s})$ & $17.1(5.0)$ & $17.2(5.8)$ & $23.3(6.5)$ & $20.5(6.6)$ \\
\hline$D_{\text {RAO }}(\mathrm{mm})$ & $1.7(0.3)$ & $1.8(0.2)$ & $2.3(0.2)$ & $2.3(0.2)$ \\
\hline $\mathrm{D}_{\text {LAO }}(\mathrm{mm})$ & $1.7(0.2)$ & $1.7(0.2)$ & $2.3(0.3)$ & $2.3(0.3)$ \\
\hline CSA $\left(\mathrm{mm}^{2}\right)$ & $2.4(0.6)$ & $2.4(0.6)$ & $4.1(0.8)$ & $4.1(0.8)$ \\
\hline $\mathrm{CBF}(\mathrm{ml} / \mathrm{min})$ & $9.1(3.0)$ & $9.0(3.2)$ & $23.8(10.7)$ & $22.8(10.4)$ \\
\hline
\end{tabular}

Values are mean (SD).

ADPV, average diastolic peak velocity; APV, average peak velocity; ASPV, average systolic peak velocity; BP, blood pressure; CBF, local coronary blood flow; $C S A$, cross sectional area; $D_{R A O}$ and $D_{\perp A O}$, vessel diameter $5 \mathrm{~mm}$ distal to the tip of the flow wire in right anterior oblique and left anterior oblique projections; glib, glibenclamide. 


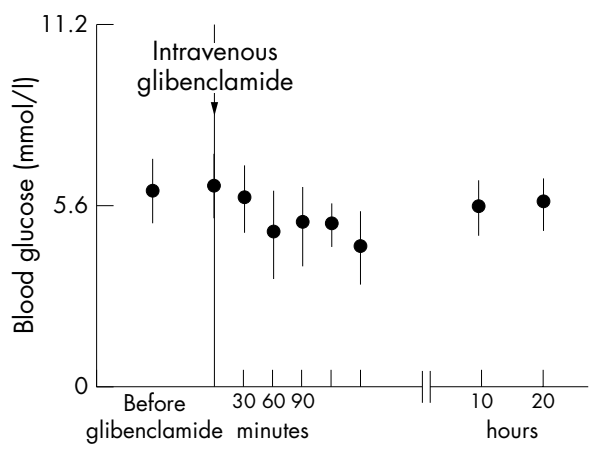

Figure 4 Blood glucose concentrations directly before administration of glibenclamide and during follow up. Values are means, error bars $=\mathrm{SD}$.

pressure remained constant during the experimental procedure and no significant changes were observed after administration of glibenclamide (table 3 ).

\section{Serum insulin, $\mathrm{C}$ peptide, and blood glucose}

Serum insulin increased significantly one hour after administration of glibenclamide, $0.05 \mathrm{mg} / \mathrm{kg}$ body weight $(\mathrm{p}<0.005)$ (fig 3). C peptide concentrations also increased significantly over the same time period (fig 3). There was only a slight decrease in blood glucose during the procedure (fig 4), as 5\% glucose was given continuously intravenously. Blood glucose concentrations were monitored for 24 hours. No patient had clinical symptoms of hypoglycaemia during follow up.

\section{DISCUSSION}

The main results of this invasive study in patients with coronary artery disease were as follows:

- coronary blood flow before and after administration of $0.05 \mathrm{mg} / \mathrm{kg}$ glibenclamide was not significantly different in post-stenotic and angiographically normal coronary arteries

- intravenous glibenclamide at a dose of $0.05 \mathrm{mg} / \mathrm{kg}$ body weight increased serum insulin and $\mathrm{C}$ peptide concentrations significantly

- the amount of coronary vasodilatation induced by adenosine and papaverine was not significantly different before and after administration of glibenclamide

\section{Coronary flow and $\mathrm{K}_{\mathrm{ATP}}$ channels in animal studies}

Recent studies on different animal models have provided a large body of knowledge about the mechanisms responsible for the regulation of coronary blood flow in different conditions, such as hypoxia, ischaemia, coronary autoregulation, and increased metabolic demand.

First described by Daut and colleagues in 1990, ${ }^{3}$ hypoxic coronary vasodilatation is mediated by opening of the vascular $\mathrm{K}_{\text {AтP }}$ channels in the isolated saline perfused guinea pig heart. Apart from the myogenic and flow induced vascular control of basal coronary tone, it has been suggested that $\mathrm{K}_{\text {AтP }}$ channels play an important role in maintaining basal coronary flow. ${ }^{572}{ }^{16}$ A significant proportion of the vasodilator action of adenosine is also mediated by the activation of $\mathrm{K}_{\mathrm{ATP}}$ channels in different models. ${ }^{37}$ As shown by Duncker and colleagues, ${ }^{6}{ }^{18}$ synergism between endogenous adenosine and opening of the $\mathrm{K}_{\text {ATP }}$ channels is important in maintaining coronary flow during exercise both in normal hearts and distal to an artificial coronary stenosis in dogs. Studies of the contribution to coronary flow of $\mathrm{K}_{\text {ATP }}$ channel activation, adenosine, and nitric oxide suggest the nearly complete suppression of nitric oxide production in hypoxia but an important role for $\mathrm{K}_{\text {ATP }}$ channels and adenosine in determining coronary tone during the different phases of hypoxia. ${ }^{4}{ }^{19}$ In dogs, adenosine receptor blockade and nitric oxide synthase inhibition do not impair the normal increase in coronary flow during exercise. However, following blockade of the $\mathrm{K}_{\text {ATP }}$ channel system, both adenosine and nitric oxide seemed to contribute to the exercise induced increase in blood flow. ${ }^{20}$ Other studies have shown that the regulation of coronary tone during metabolic stimulation, pacing, and exercise depends mainly on nitric oxide and not on $\mathrm{K}_{\text {ATP }}$ channel activation. ${ }^{21-23}$

In the present study, coronary blood flow in the angiographically normal coronary artery can be compared with investigations on basal coronary tone in animal studies. However, a simple transfer of animal models to a clinical situation in human patients is not feasible. Several animal studies have been performed on isolated hearts perfused with saline at constant pressure. In such studies, the role of the $\mathrm{K}_{\text {ATP }}$ channel in the coronary circulation might not be completely comparable because of perfusion pulsatility, the effects of the type of perfusion medium, denervation of the heart, and alterations of neurohumoral interactions. As recently shown in a dog model, glibenclamide lowered regional coronary flow under basal conditions, but with higher perfusion pulsatility no reduction in regional coronary flow was observed during glibenclamide administration. ${ }^{24}$

Post-stenotic coronary flow in human patients represents a situation of impaired oxygen supply, but might be best described as a chronic adaptation to a reduced perfusion pressure. In a closed chest model in dogs, coronary autoregulation (defined as the ability to maintain constant flow under conditions of changing perfusion pressure) was not affected by glibenclamide in a range between $60-100 \mathrm{~mm} \mathrm{Hg} .{ }^{25}$ Animal models of pure hypoxia and increased metabolic stimulation differ from the clinical setting of chronic coronary stenosis with respect to the regulation of coronary flow.

\section{Vascular $K_{\text {ATP }}$ channels in humans}

Despite intense animal research, the role of vascular $K_{\text {AтP }}$ channels in the human coronary circulation is still under debate. The forearm vasodilator response to diazoxide (a $\mathrm{K}_{\text {AтP }}$ channel opener) - as measured by venous occlusion plethysmography in healthy volunteers-was inhibited by glibenclamide. ${ }^{26}$ It was concluded that therapeutic concentrations of glibenclamide result in a significant blockade of vascular $\mathrm{K}_{\text {AтP }}$ channels in humans. Similarly, the vasodilator response to ischaemia in the forearm was reduced by glibenclamide. ${ }^{11}$ However, there is only limited information about the role of $\mathrm{K}_{\text {ATP }}$ channels in the human coronary circulation. In a combined angiographic and intracoronary Doppler study, post-stenotic blood flow was increased after intracoronary application of nicorandil, a $\mathrm{K}_{\text {AтP }}$ channel opener with additional effects similar to those of nitrates. ${ }^{27}$

In the present study, the influence of a therapeutic dose of glibenclamide on coronary flow was investigated in patients with coronary artery disease. Measurements of serum insulin and $\mathrm{C}$ peptide concentrations after administration of glibenclamide revealed a pronounced effect on insulin release, suggesting an inhibitory effect on pancreatic $\mathrm{K}_{\text {ATP }}$ channels which results in enhanced exocytic insulin release-as expected from the pharmakokinetic data on glibenclamide. ${ }^{28}{ }^{29}$ Concentrations of sulfonylurea required to block vascular $\mathrm{K}_{\text {ATP }}$ channels may be greater than those effective at the pancreatic $\beta$ cells. ${ }^{30}$ Therapeutic doses of glibenclamide possibly inhibit a fraction of $\mathrm{K}_{\text {ATP }}$ channels in coronary smooth muscle cells and might impair hypoxic coronary vasodilatation, as suggested by the apparent dissociation constants of glibenclamide at vascular and pancreatic $K_{\text {ATP }}$ channels in guinea pigs. ${ }^{5}$ Our present study suggests that coronary basal tone in angiographically normal coronary arteries, post-stenotic coronary flow at rest, and the hyperaemic response to adenosine are not significantly altered after therapeutic doses of glibenclamide. 


\section{Coronary flow assessed by Doppler flow profiles and quantitative angiography}

Determination of coronary flow characteristics using intracoronary Doppler measurements of coronary flow velocity in combination with quantitative angiographic analysis is a well established method of investigating coronary blood flow in vivo. Validation studies have shown a high correlation in experimental animal studies ${ }^{14}$ between ${ }^{133} \mathrm{Xe}$ perfusion studies, ${ }^{31}{ }^{15} \mathrm{O}-\mathrm{H}_{2} \mathrm{O}$ myocardial positron emission tomography, ${ }^{32}$ and continuity equation calculations. ${ }^{33}$ The estimated mean blood flow velocity was calculated as $0.5 \times$ average peak velocity by assuming a time averaged parabolic velocity profile across the vessel. ${ }^{15}$ Using a combination of Doppler flow measurements and cross sectional intravascular ultrasound imaging, other groups have suggested a better correlation with a calculated mean flow velocity of $0.47 \times$ average peak velocity. ${ }^{34}$ Assessment of coronary flow reserve from average peak velocity after papaverine and adenosine is a well established method, but it might underestimate the hyperaemic response, as changes of vessel diameters are not taken into account by this method. ${ }^{35}{ }^{36}$

\section{Sulfonylurea drugs and coronary artery disease}

The potential risk of sulfonylurea drug treatment in diabetic patients with coronary artery disease is still under debate. In a prospective trial in 3867 newly diagnosed diabetic patients, there was no difference in the clinical outcome after 10 years between sulfonylurea treatment and insulin treatment. ${ }^{37}$ On the other hand, some clinical studies have suggested that there is an increased cardiovascular mortality in patients on sulfonylurea drugs. ${ }^{8}{ }^{98}$ These effects have mainly been attributed to the inhibitory actions of sulfonylurea drugs on preconditioning-like cardioprotective effects. In the human model of ischaemic preconditioning during angioplasty, the degree of ST segment shift during balloon inflation is reduced during the second balloon inflation compared with the first. This preconditioning-like effect is prevented by glibenclamide and thus could be an expression of the same effect that is responsible for the increased cardiovascular mortality in sulfonylurea drug treatment. ${ }^{39} 40$

A deterioration in coronary blood supply is an alternative explanation but has not so far been investigated in humans. In our present study no significant impairment of coronary blood flow was observed after administration of a therapeutic dose of glibenclamide. Nevertheless, significant effects on coronary haemodynamics and collateral circulation under conditions of exercise or electrical pacing, acute myocardial ischaemia, left ventricular hypertrophy, or in diabetic microvascular disease with impairment of nitric oxide mechanisms cannot be excluded. The results of the present study do not necessarily apply to diabetic patients, to patients with acute myocardial ischaemia, or in particular to patients with microvascular disease. The coronary flow reserve in the angiographically normal artery was above the level of 2.0 in all patients, suggesting no significant microvascular disease in this population, and no patient was diabetic. These are major limitations of the study that warrant further investigation.

\section{Conclusions}

Alternatives to sulfonylurea drugs should be considered carefully in the treatment of diabetic patients with coronary artery disease, despite there being no deterioration in coronary flow at rest under the influence of glibenclamide in this population. ${ }^{130}$

\section{Authors' affiliations}

T Reffelmann, H G Klues, P Hanrath, E R Schwarz, Medizinische Klinik I, University Hospital, Rheinisch-Westfälische Technische Hochschule (RWTH), Pauwelsstrasse 30, D-52057 Aachen, Germany

\section{REFERENCES}

1 Engler RL, Yellon DM. Sulfonylurea $K_{\text {ATP }}$ blockade in type II diabetes and preconditioning in cardiovascular disease time for reconsideration. Circulation 1996:94:2297-301.

2 Boyd AE, Aguilar-Bryan L, Nelson DA. Molecular mechanisms of action of glyburide on the beta cell. Am J Med 1990;89:3-10.

3 Daut J, Maier Rudolph W, von Beckerath N, et al. Hypoxic dilation of coronary arteries is mediated by ATP-sensitive potassium channels. Science 1990:247:1341-4.

4 Kamekura I, Okumura K, Matsui H, et al. Mechanisms of hypoxic coronary vasodilation in isolated perfused rat hearts. J Cardiovasc Pharmacol 1999;33:836-42.

5 Daut J, Klieber HG, Cyrus S, et al. $\mathrm{K}_{\text {ATP }}$ channels and basal coronary vascular tone. Cardiovasc Res 1994:28:811-17.

6 Duncker DJ, van Zon NS, Ishibashi Y, et al. Role of K+ ATP channels and adenosine in the regulation of coronary blood flow during exercise with normal and restricted coronary blood flow. J Clin Invest 1996;97:996-1009.

7 Dellsperger KC. Potassium channels and the coronary circulation. Clin Exp Pharmacol Physiol 1996:23:1096-101.

$8 \mathrm{Klimt}$ CR, Knatterud GL, Meinert CL, et al. A study of the effect of hypoglycemic agents on vascular complications in patients with adult-onset diabetes. Diabetes 1970;19:747-830.

9 Garrat KN, Brady PA, Hassinger NL, et al. Sulfonylurea drugs increase early mortality in patients with diabetes mellitus after direct angioplasty for acute myocardial infarction. J Am Coll Cardiol 1999:33:1 19-24.

10 Schwarz ER, Reffelmann T, Kloner RA. Clinical effects of ischemic preconditioning. Curr Opin Cardiol 1999;14:340-8.

11 Bijlstra PJ, den Arend JA, Lutterman JA, et al. Blockade of vascular ATP-sensitive potassium channels reduces vasodilator response to ischemia in humans. Diabetologia 1996:39:1562-8.

12 Cyrys S, Daut J. The sensitivity of coronary vascular tone to glibenclamide: a study on the isolated perfused guinea pig heart. Cardiovasc Res 1994;28:888-93.

13 von Birgelen C, Kutryk M, Gil R, et al. Quantification of the minimal luminal cross-sectional area after coronary stenting by two- and three dimensional intravascular ultrasound versus edge detection and videodensitometry. Am J Cardiol 1996;78:520-5

14 Brown BG, Bolson E, Frimer M, et al. Quantitative coronary angiography. Circulation 1977:55:329-37.

15 Doucette JW, Corl PD, Payne HM, et al. Validation of a Doppler guide wire for intravascular measurement of coronary artery flow velocity. Circulation 1992;85:1899-911.

16 Komaru T, Kanatsuka H, Shirato K. Coronary microcirculation. Physiology and pharmacology. Pharmacol Ther 2000;86:217-61

17 Randall MD. The involvement of ATP-sensitive potassium channels and adenosine in the regulation of coronary flow in the isolated perfused rat heart. Br J Pharmacol 1995; 1 16:3068-74.

18 Duncker DJ, van Zon NS, Pavek TJ, et al. Endogenous adenosine mediates coronary vasodilation during exercise after K(ATP)+ channel blockade. J Clin Invest 1995;95:285-95.

19 Decking UKM, Reffelmann T, Schrader J, et al. Hypoxia-induced activation of $\mathrm{K}_{\mathrm{ATP}}$ channels limits energy depletion in the guinea pig heart. Am J Physiol 1995;269:H734-42.

20 Ishibashi Y, Duncker DJ, Zhang J, et al. ATP-sensitive K+ channels, adenosine, and nitric oxide mediated mechanisms account for coronary vasodilation during exercise. Circ Res 1998:82:346-59.

21 Embrey RP, Brooks LA, Dellsperger KC. Mechanism of coronary microvascular responses to metabolic stimulation. Cardiovasc Res 1997;35: 148-57

22 Richmond KN, Tune JD, Gorman MW, et al. Role of K(ATP) $(+)$ channels and adenosine in the control of coronary blood flow during exercise. $J$ Appl Physiol 2000;89:529-36

23 Richmond KN, Tune JD, Gorman MW, et al. Role of K+ATP channels in local metabolic coronary vasodilation. Am J Physiol 1999;277:H2 1 15-23.

24 Pagliaro P, Paolocci N, Isoda T, et al. Reversal of glibenclamide-induced coronary vasoconstriction by enhanced perfusion pulsatility: possible role for nitric oxide. Cardiovasc Res 2000:45:1001-9.

25 Stepp DW, Kroll K, Feigl EO. K+ATP channels and adenosine are not necessary for coronary autoregulation. Am J Physiol 1997;273:H1299-308.

26 Bijlstra PJ, Lutterman JA, Russel FG, et al. Interaction of sulfonylurea derivates with vascular ATP-sensitive potassium channels in humans. Diabetologia 1996;39:1083-90

27 Haager PK, Klues HG, Schmidt M, et al. Effect of nitroglycerin and nicorandil on regional poststenotic quantitative coronary blood flow in coronary artery disease: a combined digital quantitative angiographic and intracoronary Doppler study. J Cardiovasc Pharmacol 1999;33:126-34

28 Neugebauer G, Betzien G, Herstka V, et al. Absolute bioavailability and bioequivalence of glibenclamide (Semi-Euglucon N). Int J Clin Pharmacol Ther Toxicol 1985;23:453-60.

29 Ings RMJ, Lawrence JR, McDonald A, et al. Glibenclamide pharmacokinetics in healthy volunteers: evidence for zero-order drug absorption. Br J Clin Pharmacol 1982;13:264-5

30 Connaughton $M$, Webber J. Diabetes and coronary artery disease: time to stop taking the tablets? Heart 1998;80:108-9

31 Dib N, Bajwa T, Shalev Y, et al. Validation of Doppler FloWire for measurement of coronary flow reserve in humans. Cathet Cardiovasc Diagn 1998;45:382-5

32 Miller DD, Donohue TJ, Wolford TL, et al. Assessment of blood flow distal to coronary artery stenoses. Correlations between myocardial positron emission tomography and poststenotic intracoronary Doppler flow reserve. Circulation 1996;94:2447-54 
33 Doriot P, Dorsaz P, Dorsaz L, et al. Accuracy of coronary flow measurements performed by means of Doppler wires. Ultrasound Med Biol 2000;26:221-8

34 Chou TM, Sudhir K, Iwanaga S, et al. Measurement of volumetric coronary blood flow by simultaneous intravascular two-dimensional and Doppler ultrasound: validation in an animal model. Am Heart $J$ 1994; 128:237-43.

35 Takeuchi $M$, Nohtmi Y, Kuroiwa A. Does coronary flow reserve assessed by blood flow velocity analysis reflect absolute coronary flow reserve? Cathet Cardiovasc Diagn 1996;38:251-4.

36 Kaufmann PA, Jenni R. Coronary flow reserve assessment from average peak velocity profiles alone must be judged with caution. J Am Coll Cardiol 2000;35:1363-5.
37 UK Prospective Diabetes Study (UKPDS) Group. Intensive blood-glucose control with sulfonylureas or insulin compared with conventional treatment and risk of complications in patients with type 2 diabetes (UKPDS 33). Lancet 1998;352:837-53.

38 Rytter L, Troeter S, Beck-Nielsen H. Prevalence and mortality of acute myocardial infarction in patients with diabetes. Diabetes Care $1985: 8: 230-4$

39 Tomai F, Crea F, Gaspardone A, et al. Ischemic preconditioning during coronary angioplasty is prevented by glibenclamide, a selective ATP-sensitive K+ channel blocker. Circulation 1994,90:700-5.

40 Klepzig $\mathbf{H}$, Kober G, Matter $\mathrm{C}$, et al. Sulfonylurea and ischaemic preconditioning. A double-blind, placebo-controlled evaluation of glimepiride and glibenclamide. Eur Heart J 1999;20:439-46.

\section{IMAGES IN CARDIOLOGY}

\section{Localised cardiac tamponade caused by intrapericardial haematoma: a rare cause of ascites presenting 10 years after open heart surgery}

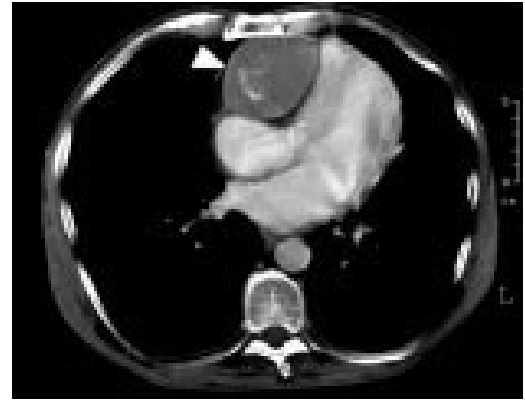

59 year old man underwent quadruple coronary artery bypass grafting in 1989. Ten years later he was admitted to hospital as an emergency with a two week history of increased abdominal swelling, lethargy, breathlessness and ankle swelling. Examination revealed a sinus tachycardia, raised jugular venous pressure, pronounced abdominal ascites, and pitting oedema of the ankles. He was normotensive with normal heart sounds and no significant murmurs. His lung fields were clear. Transthoracic echocardiography revealed moderate left ventricular systolic impairment and localised right ventricular tamponade. Computed tomography confirmed significant compression of the right ventricle by a pericardial mass which contained some central calcification. No communication with the cardiac chambers or extrinsic structures could be identified. At surgery the mass was successfully excised. Histological analysis revealed an organised, partly calcified haematoma. Cardiac tamponade after surgery most often occurs within the first postoperative week or two and generally presents acutely with haemodynamic compromise. Intrapericardial organised haematoma, causing localised compression of the cardiac chambers late after open heart surgery, is unusual.
N D Palmer

J Curtis

E A Rodrigues

nickpalmer@supanet.com

\section{Transcatheter closure of coronary artery to pulmonary artery fistula using covered stents}

$A^{4}$ 40 year old normotensive, diabetic man presented to the emergency department in congestive heart failure. He had been diagnosed elsewhere with dilated cardiomyopathy. The ECG revealed $\mathrm{T}$ wave inversion in precordial leads, and echocardiography showed dilated left ventricle (LV) with global hypokinesia and severe LV dysfunction. ${ }^{99 m}$ Tc labelled RBC MUGA showed an enlarged LV with ejection fraction of $23 \%$, and ${ }^{99 m}$ Tc-sestamibi resting myocardial perfusion study revealed reduced tracer concentration in the anterior wall and septum and viable myocardium in these areas. A subsequent coronary angiogram showed multiple coronary fistulas from the septal branches of the left anterior descending coronary artery (LAD) draining into the pulmonary artery (below left). A $3 \times 19 \mathrm{~mm}$ Jostent coronary stent graft was deployed in the LAD to occlude the septal arteries feeding the fistulas. As the post deployment angiogram (below centre) showed residual fistulas from the septal branches distal to the stent, another $3 \times 12 \mathrm{~mm}$ Jostent was deployed in the distal part of the first stent occluding the septals causing residual fistulas The final angiogram showed good antegrade flow in the LAD with obliteration of fistulas (below right).

Coronary fistulas most commonly originate from the right coronary artery and the majority are asymptomatic. The related problems that occur usually are myocardial ischaemia and angina (the result of a "coronary steal"), congestive heart failure, bacterial endocarditis, cardiac arrhythmia or rupture of an aneurysmal fistula. Current treatment options include surgical ligation and coil embolisation. Recently covered stents have been successfully employed for the closure of coronary fistulas.

A S Mullasari

C V Umesan K Jagadeesh Kumar icvd@eth.net
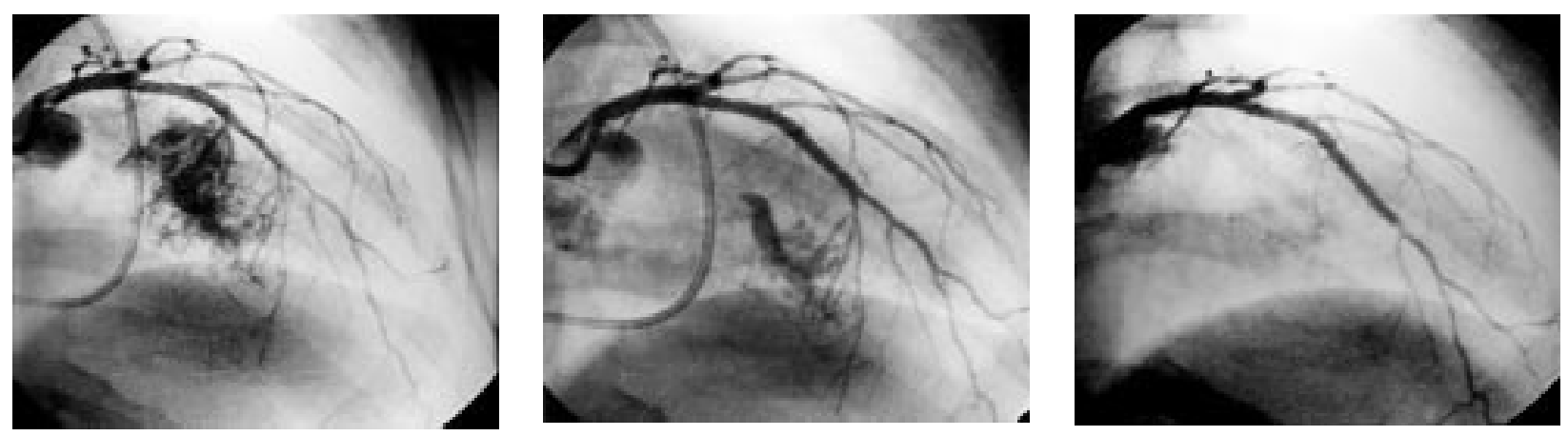